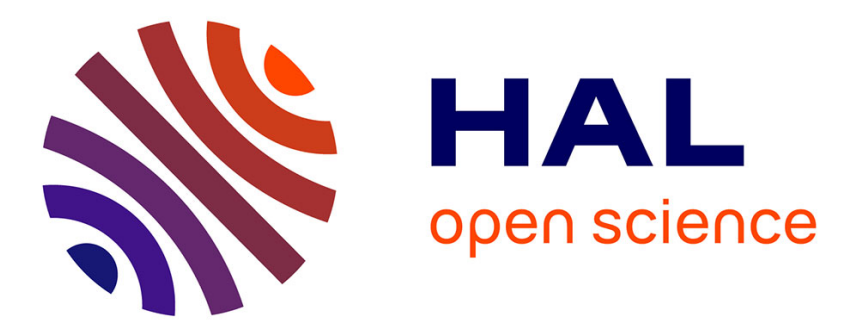

\title{
Experimental and theoretical investigation of the Preglow in ECRIS
}

I.V. Izotov, T. Lamy, L. Latrasse, A.V. Sidorov, V.A. Skalyga, T. Thuillier, V.G. Zorin

\section{- To cite this version:}

I.V. Izotov, T. Lamy, L. Latrasse, A.V. Sidorov, V.A. Skalyga, et al.. Experimental and theoretical investigation of the Preglow in ECRIS. IEEE Transactions on Plasma Science, 2008, 36/4, pp.14941501. 10.1109/TPS.2008.927292 . in2p3-00193403v2

HAL Id: in2p3-00193403 https://hal.in2p3.fr/in2p3-00193403v2

Submitted on 14 Oct 2008

HAL is a multi-disciplinary open access archive for the deposit and dissemination of scientific research documents, whether they are published or not. The documents may come from teaching and research institutions in France or abroad, or from public or private research centers.
L'archive ouverte pluridisciplinaire HAL, est destinée au dépôt et à la diffusion de documents scientifiques de niveau recherche, publiés ou non, émanant des établissements d'enseignement et de recherche français ou étrangers, des laboratoires publics ou privés. 


\title{
Experimental and Theoretical Investigation of the Preglow in ECRIS
}

\author{
V. Izotov $^{(1)}$, A. V. Sidorov ${ }^{(1)}$, V. A. Skalyga ${ }^{(1)}$, V. G. Zorin ${ }^{(1)}$, T. Lamy ${ }^{(2)}$, L. Latrasse $^{(2)}$, T. Thuillier ${ }^{(2)}$
}

(1) Institute of Applied Physics of Russian Academy of Sciences, 46 Ulyanov Street, 603950, Nizhny Novgorod, Russia.

(2) Laboratoire de Physique Subatomique et de Cosmologie, Université Joseph Fourier Grenoble 1, CNRS/IN2P3, INP Grenoble, Grenoble, France.

\begin{abstract}
The experimental study of pulsed mode operation of the PHOENIX-V2 Electron Cyclotron Resonance Ion Source at $28 \mathrm{GHz}$ has clearly demonstrated, when increasing the repetition rate of the HF power injection at frequencies higher than $1 \mathrm{~Hz}$, the reality of a transient current peak occurring at the very beginning of the plasma discharge. This regime was named the Preglow, as an explicit reference to the classical Afterglow occurring at the microwave pulse end. After the transient Preglow peak, the plasma regime relaxes to the classical steady state one. Experimental argon pulses for charge states from $2+$ to $8+$ are presented. The current observed during the preglow peak can reach intensities of the order of $1 \mathrm{~mA}$ for low charge states $(\mathrm{Ar} 4+)$. A 0-dimension theoretical model of ECR gas breakdown in a magnetic trap is presented in detail. Results of the simulation are compared with the experimental Preglow peaks and discussed.
\end{abstract}

\section{INTRODUCTION}

ELECTRON Cyclotron Resonance Ion Sources (ECRIS) can be used in continuous or pulsed operation. The pulsed operation, permitting to generate the well known Afterglow mode (AFG) [1, 2], has been used daily at CERN for pulsed heavy ion beams production [3]. When varying the parameters (confinement magnetic field, microwave frequency and power, gas pressure, gas nature ...), different plasma regimes can be observed. If one can find stable and reproducible regimes, new applications for ECRIS may be found. For example, within the EURISOL design study, the Beta Beam project [4] may take profit of direct, fast and efficient multi ionization of $6 \mathrm{He}$ and $18 \mathrm{Ne}$ gases. The generation of $2 \times 1013$ ions of $6 \mathrm{He} 2+$ or $8 \times 1011$ ions of $18 \mathrm{Ne}^{+}$, grouped in a 50 to $100 \mu \mathrm{s}$ pulse, would permit a direct injection into a post accelerator LINAC. When injecting pulsed HF power with a repetition rate of $1 \mathrm{~Hz}$ into the PHOENIX-V2 ECRIS, it can be noticed a fast pressure decrease leading to instabilities during the pulse. This fast pressure decrease is associated with a fast transient intensity peak occurring at the beginning of the plasma discharge. Due to the high drain current of the high voltage supply during this peak, it is supposed that the pressure drop is the consequence of the ionic pumping and that the instabilities are due to the inadequate HF coupling in such low plasma density. This peak has been named Preglow mode (PGW) [5] in analogy with the AFG which occurs at the microwave pulse end. To generate such a peak in experiments, it appears to be necessary to operate with high repetition rates (typically $1 \mathrm{~Hz}$ ). In such experimental conditions, a low density plasma with hot electrons can be confined from pulse to pulse if its lifetime is higher than the delay between HF pulses. The presence of this low density plasma at the very beginning of the RF pulse seems to be an important condition to generate PGW.

This paper is organized as follows: in the first section the experimental setup is described and an example of experimental argon PGW pulses are presented and quantitatively described. After a second section, where a theoretical 0-dimension simulation program intending to explain experimental data is presented, the simulation results are presented and compared with the experimental ones leading to a global discussion and to the conclusion.

\section{EXPERIMENTAL DESCRIPTION}

The experiments have been performed on the PHOENIX-V2 ECRIS test bench described in details in [6]. The PHOENIX-V2 source is a modified version of the original PHOENIX one. The radial confinement magnetic field has been improved and the length of the hexapole reduced from $320 \mathrm{~mm}$ to $240 \mathrm{~mm}$. The plasma chamber volume is $\sim 0.7$ liter. The minimum- $|\mathrm{B}|$ structure of the ECRIS is obtained by the superposition of a radial hexapolar field $(\mathrm{Br}=1.3$ Tesla at the plasma chamber walls) with an axial magnetic field forming a magnetic bottle tunable within the following ranges: at the injection side the maximum magnetic induction is Bzinj $\leq 1.66 \mathrm{~T}$, in the middle plane $0.45 \mathrm{~T} \leq$ Bzmid $\leq 0.95 \mathrm{~T}$, and at the extraction side Bzextr $\leq 1.31 \mathrm{~T}$. The plasma is heated with a $28 \mathrm{GHz}$ frequency microwave delivered by a Gycom gyrotron [7] with a $10 \mathrm{~Hz}$ repetition rate and a microwave pulse duration in the range of 5 to $15 \mathrm{~ms}$. The total beam extracted from the ion source is analyzed through a $90^{\circ}$ magnetic spectrometer and collected in a Faraday cup. The plasma electrode hole has a diameter of $10 \mathrm{~mm}$ and the extraction voltage is ranging from 30 to 40 $\mathrm{kV}$. These values were chosen to get good beam transmission efficiency $(>80 \%)$ throughout the $90^{\circ}$ dipole 
to the analysis Faraday cup. The high transmission efficiency permits to consider that the ionic spectra obtained give a confident picture of the ECRIS plasma boundary conditions where the ions are extracted from.

The technique used to analyze the experimental ionic pulses is described in [8]. A typical time evolution of the multi-charged argon intensities for different charge states is presented on Fig. 1, where the PGW peaks for charge states from $2+$ to $8+$ can be seen. The HF pulse begins at $t=0$ and ends at $t=10 \mathrm{~ms}$, the spectrum is optimized on the Ar4+ PGW peak due to a high gas flow used for the experiment. On the vertical scale the intensity between two sticks is $500 \mu \mathrm{A}$, the signals have been shifted up by this value for better view.

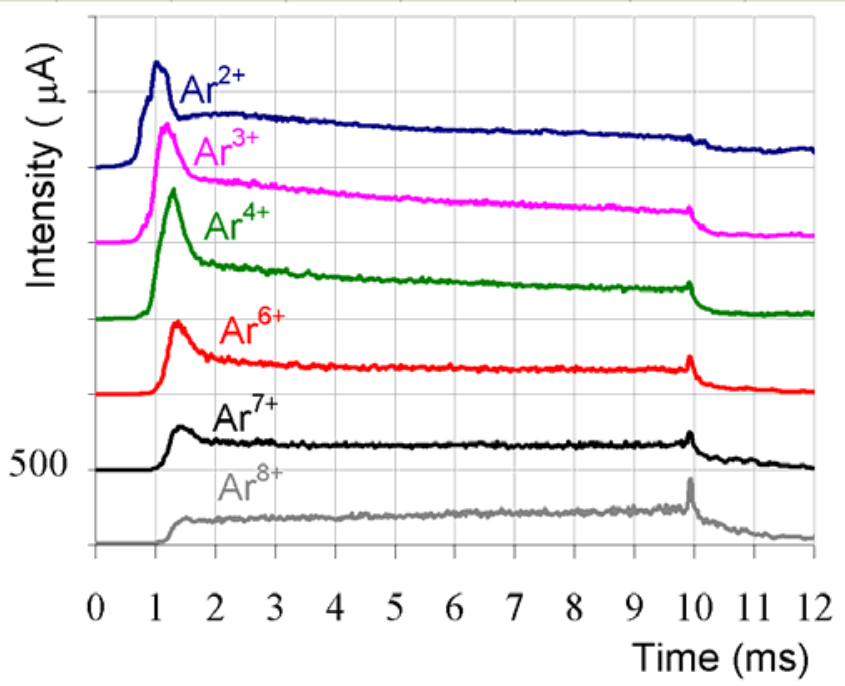

Fig. 1. Example of experimental PGW peaks for argon. The microwave power is $3100 \mathrm{~W}$, the extraction voltage is $30 \mathrm{kV}$, the pulse duration is $10 \mathrm{~ms}$ and the plasma electrode diameter is $10 \mathrm{~mm}$.

The PGW peaks, whatever is the charge state, appear in the time range from $\sim 1$ to $\sim 1.5 \mathrm{~ms}$, this implies a fast ionization process with a hot and dense electron population. After $2 \mathrm{~ms}$, the steady state is reached for every charge state, and at the end of the HF pulses (10 ms) the AFG peak increases for increasing charges. If we count the number of ions in a time window of $100 \mu$ s centered on the PGW peak maximum for each charge state, we see that the maximum number is available on the $\mathrm{Ar}^{2+}$, as shown in Fig. 2.

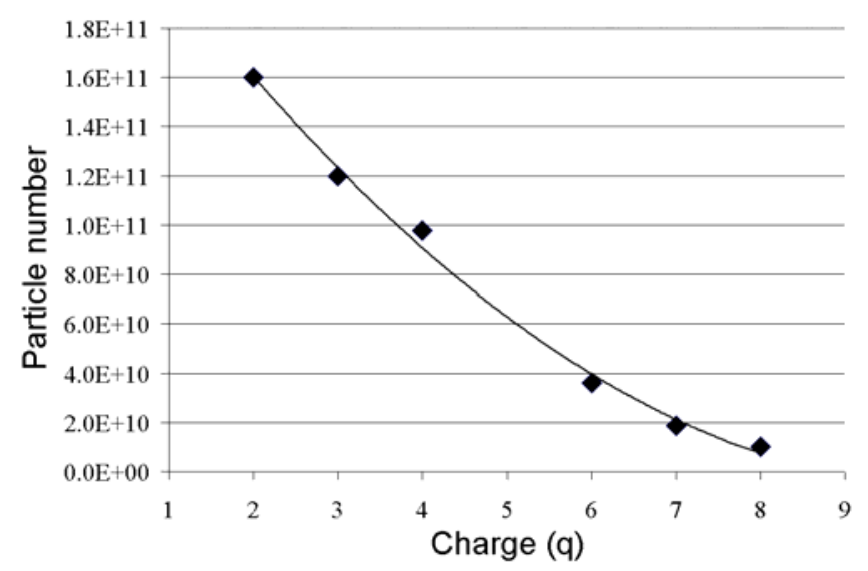

Fig. 2. Number of ions in a $100 \mu$ s time window centered on the PGW peak maximum for each charge state.

\section{THEORETICAL PLASMA MODEL}

In order to have a good understanding of the plasma characteristics evolution, a detailed analysis of the gas discharge ignition is necessary. The following description of PGW phenomenon is based on calculations which have been made with the zero- dimension plasma confinement model in a magnetic trap, which was firstly proposed in [9]. Significant additions to this model and calculations for different argon charge states have been performed and are presented here.

A microwave induced plasma ignition of a rarefied gas in a magnetic trap under the ECR conditions may be separated into two stages for which the growth rate of the plasma density is determined by different processes 
governed by the evolution of the electron energy distribution function (EEDF). At the first stage, the density is low and the electrons undergo a fast energy increase, the dominating process is the neutral gas ionization due to collisions with hot electrons. The plasma density at this stage grows fast, the gas ionization degree is low and the power absorbed by the plasma is less than the one absorbed at the steady state of the discharge. At the second stage, the increase of the density slows down significantly, the step by step ionization process goes further modifying the EEDF, the average charge state increases, and the power absorbed by the plasma is approximately equal to the value at the steady state.

The initial stage of the plasma ignition, when the density is sufficiently low, can be described with the theory for a microwave breakdown of a rarefied gas in axi-symmetric mirror magnetic traps under the electron cyclotron resonance conditions [10]. In this work, an important factor which significantly affects the EEDF shape is the superadiabaticity effect. When the plasma density is so low that the electron collision time is much longer than the interaction time of an electron with the HF wave in the resonance region, the energy transfer can be described like for a single electron. The ECR energy increase of the lone electron in a mirror trap is governed by stochasticity, and this remains true when the phase change of the microwave between two electron passes through the ECR zone is much more than $2 \pi[10]$. With the electron energy increase, the electron travel time from mirror to mirror decreases and consequently the phase variation of the heating wave decreases too, this means that at a certain electron energy value the stochastic mechanism of electron heating is destructed. At this stage the interaction of hot electrons with a microwave is called superadiabatic interaction. The consequence of this interaction is an electron energy limitation which is called superadiabaticity effect. Hence, while the plasma density is low, the plasma is unable to absorb more energy than a certain value, because the energy of every electron is limited. In [10] it has been shown that the following EEDF is formed under such conditions:

$F=\frac{N_{e 0}}{2}\left(\sqrt{\varepsilon \varepsilon_{s t}}\right)^{-1} 1\left(\varepsilon_{s t}-\varepsilon\right) \exp \left(v_{e f f} t\right)(1)$

where $1(\mathrm{x})$ is the unit Heaviside function, $N_{e 0}$ the initial number of electrons, $\varepsilon_{s t} \propto \omega^{1 / 2} E^{3 / 4}$ the energy boundary value determined by the superadiabaticity effect ( $\omega$ : HF frequency, $E$ : electric field amplitude of the wave), $v_{\text {eff }}=\left(\tau^{*}\right)^{-1}$ where $\tau^{*}$ is the electron density increase characteristic time. In the case of axi-symmetric magnetic traps, as it has been analytically shown in [10], the formation time of the EEDF (1) is much shorter than $\tau^{*}$, the EEDF obtained was later confirmed by numerical calculations performed with the General ECRIS Model code developed by FARTECH, Inc. and based on a 1D bounce-averaged Fokker-Planck modeling for the electrons [11]. An example of such a superadiabatic EEDF at the very beginning of an ECR discharge is plotted in Fig. 3.

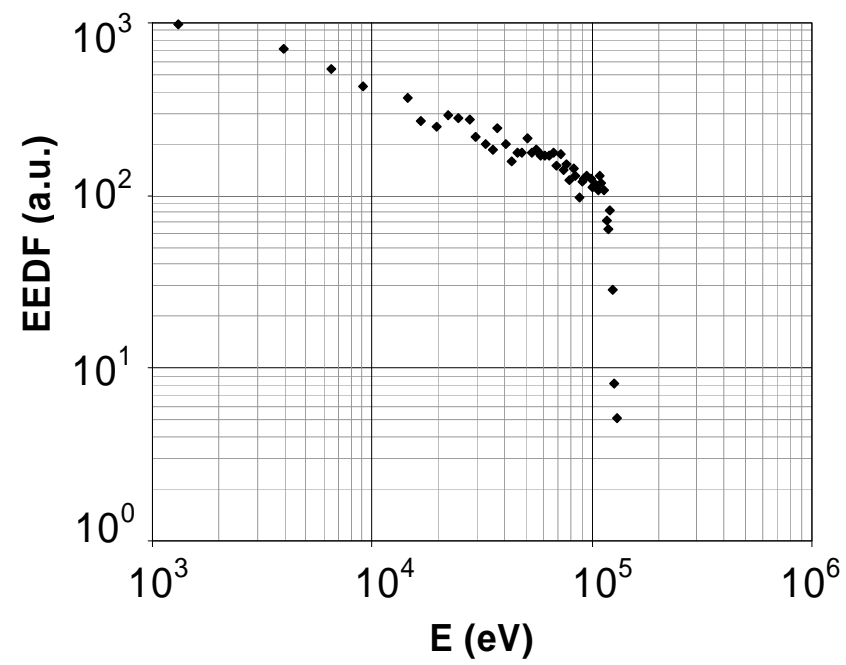

Fig. 3. Example of a superadiabatic EEDF.

When the plasma density increases further, the absorbed heating power becomes insufficient to maintain the EEDF (1). This happens when the total energy, absorbed since the beginning of the discharge, equals to the sum of the energy lost in the plasma leaks from the trap and in the ionization processes. In order to describe this 
process, it is necessary to use the electronic energy balance (equation 2) which takes into account the energy introduced by the microwave power, the energy losses caused by the plasma leaks from the trap and by the ionization processes.

$$
\frac{3}{2} \times \frac{d\left(N_{e} \times T_{e}\right)}{d t}=\frac{P}{L}-\frac{N_{e}}{\tau_{e}} \times\left(T_{e}+\Delta \psi\right)-\sum_{i=0}^{n-1} k_{i, i+1} \times N_{e} \times N_{i} \times I_{i}
$$

Here $N_{e}$ is the electron density, $T_{e}$ the electron temperature, $P$ the microwave power flow density, $L$ the trap length, $k_{i, i+1}$ the ionization rate from the charge state $i$ to the charge state $i+1, N_{i}$ the ion density for the charge $i$, $I_{i}$ the ionization potential of an ion with charge $i, \tau_{e}$ the electron life time, $\Delta \psi$ the difference between the plasma potential at the centre of the trap and behind the magnetic plug, and $n$ the electron number for the considered atom. In the framework of the considered model, since this moment the EEDF is assumed to be Maxwellian.

For modeling the plasma discharge dynamics, the energy balance equation and a non-stationary differential equations system for the ionization balance are used. The ionization balance is described by the following set of equations for all ionization states $i$ :

$$
\frac{\partial N_{i}}{\partial t}=\left(k_{i-1, i} N_{i-1}-k_{i, i+1} N_{i}\right) \cdot N_{e}-\frac{N_{i}}{\tau_{i}}-k_{i}^{\text {ch.ex. }} N_{i} N_{o}+k_{i+1}^{\text {ch.ex. }} N_{i+1} N_{o}(3)
$$

Here $\tau_{i}$ is the ion lifetime for different charge states $i$, the charge exchange rate of ion with charge $i$ on neutrals, $k_{i, i+l}$ are the electron impact ionization rate coefficients calculated for the Maxwellian EEDF:

$$
k=<\sigma v>=\frac{\int F(\varepsilon) \sigma(\varepsilon) v(\varepsilon) d \varepsilon}{\int F(\varepsilon) d \varepsilon}(4)
$$

Here $\sigma$ is the ionization cross section, $v$ the electron velocity, $F(\varepsilon)$ the EEDF. For $\sigma$, data from Povyshev (Joint Institute for Nuclear Research, Dubna) [12] are used. The equation for the electrons is written as:

$$
\frac{\partial N_{e}}{\partial t}=N_{e} \times \sum_{i=0}^{n-1} k_{i, i+1} N_{i}-\frac{N_{e}}{\tau_{e}}(5)
$$

The neutral gas density equation is:

$$
\frac{d N_{0}}{d t}=I(t)+S\left(1-Q_{e x t r}\right)-k_{0,1} N_{0} N_{e}-\sum_{i=2}^{n} k_{i}^{c h . e x .} N_{i} N_{o}(6)
$$

where $I(t)$ is the number of gas atoms injected per cubic centimeter per second into the trap, $S$ the flux of neutrals which comes from the chamber walls due to the ion neutralization on them, $Q_{\text {extr }}$ an extraction coefficient taking in account the proportion of the ion flux extracted through the plasma electrode hole (not reaching the plasma chamber walls), including such coefficient into equations allows to model the ion pumping effect. The system is supplemented by the plasma quasi-neutrality condition:

$$
\frac{N_{e}}{\tau_{e}}=\sum_{i=1}^{n} \frac{i \times N_{i}}{\tau_{i}}(7)
$$

Equations (3), (5)-(7) do not directly depend on the shape of EEDF. The dependence on the EEDF is present in calculations of $T_{e}$ and $k_{i, i+1}$ (equations 2 and 4 ).

The previous system of equations should also be supplemented by equations for plasma lifetime, and then it is necessary to determine a confinement regime of the plasma.

A mirror-confinement regime for a plasma composed of hot electrons and cold ions crucially depends on the ordering of the characteristic timescales such as the Coulomb electron scattering into the loss-cone time $\tau_{c}=\ln$ $R / v_{\mathrm{ei}}$, the thermal ion escape time from the trap $\tau_{T}=L R / V_{T}$, and the gas dynamic confinement time $\tau_{g}=L R / V_{s}$. 
Here, $R$ is the mirror ratio, $v_{e i}$ the electron-ion Coulomb collision frequency, $V_{T}=\sqrt{T_{i} / M}$ the ion thermal velocity, $V_{s}=\sqrt{\left\langle z>T_{e} / M\right.}$ the ion sound velocity for the average charge $<_{z}>$ of the ion population, $T_{i}$ and $T_{e}$ are the ion and electron temperatures and $M$ is the ion mass. At low plasma densities, we have: $\tau_{c}>\tau_{T} \gg \tau_{g}$. Therefore, the loss-cone turns out to be essentially empty and the electron confinement time $\tau_{e} \approx \tau_{c}>>\tau_{T}$ [13]. Accordingly, to keep the plasma quasi-neutrality, an ambipolar field in the trap should delay ion escape. This is achieved by means of ambipolar potential "humps" formed in the vicinities of the magnetic plugs. In this case the ion confinement time can be roughly estimated as [14], [15]:

$\tau_{i} \approx \tau_{T} \times \exp \left(\frac{z_{i} \times e \times \Delta \varphi}{k \times T_{i}}\right)(8)$

where $\tau_{i}$ is the confinement time of an ion with charge state $z_{i}, e$ the electron charge, $k$ the Boltzmann constant, and $\Delta \varphi$ the height of the potential "hump". When the plasma density increases, the electron confinement time decreases, and at some point, drops below the ion thermal escape time from the trap. Thereafter, the potential "humps" have vanished, and ambipolar field starts contributing to ion ejection from the trap $(\Delta \varphi$ becomes negative). Due to frequent ion-ion collisions, the confinement times for different kinds of ions appear to be almost the same, and thus, equal to the electron confinement time, which is determined by Coulomb scattering if $\tau_{c}>\tau_{g}:$

$\tau_{i} \approx \tau_{e} \approx \tau_{c}(9)$

or by gas-dynamic processes if the plasma density is so high that $\tau_{c}<\tau_{g}[16]$ :

$\tau_{i} \approx \tau_{e} \approx \tau_{g}(10)$

In (9) the EVDF (Electron Velocity Distribution Function) loss-cone is still empty, while in (10) it is filled with electrons. Such regime of non-equilibrium plasma confinement was called quasi-gasdynamic confinement regime [17].

Plasma losses determined by transversal diffusion in the magnetic field were not calculated due to the complexity of a minimum- $|\mathrm{B}|$ structure. However, because it can significantly change quantitative variables of PGW, transversal lifetime was included into calculations as a free parameter, as well as the microwave power absorption rate. These free parameters were adjusted to provide the best agreement between experimental and calculated ion currents for all charge states at the steady state of the discharge. The initial density of neutrals is fixed by the initial pressure in the plasma chamber and is set to $2 \times 10^{11} \mathrm{~cm}^{-3}$.

\section{COMPARISON OF NUMERICAL AND EXPERIMENTAL RESULTS}

A. Direct Comparison of Experimental and Simulated PGW Peaks

The previous described model has been used in order to simulate experimental argon PGW peaks shown on Fig. 1. The time evolution of the ionic currents has been computed simultaneously for all argon charge states with a predefined set of free parameters. For each argon charge state, the experimental current intensity and their corresponding numerical fit are shown in Fig. 4. The best fit has been obtained with the free parameters set as follows: absorption rate $=25 \%$ and upper limit for transversal lifetime $=600 \mu$ s. There is a good agreement between numerical simulations and experimental data for charges lower than $4+$ where the PGW peak intensity is well reproduced. However these peak intensity values are overestimated for higher charges, it can be explained by the approximate calculation of the transversal ion lifetime which strongly depends on the ion charge states. In Fig. 4c) we have plotted the calculated electron energy averaged over the EEDF, after about $1 \mathrm{~ms}$ due to the transition between the superadiabatic EEDF and the Maxwellian one, the average electron energy begins to collapse. 



b) $\mathrm{Ar}^{3+}$

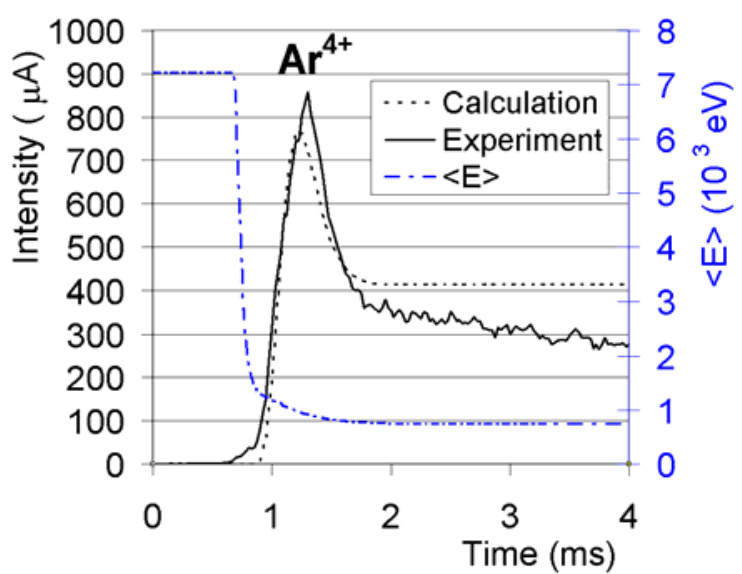

c) $\mathrm{Ar}^{4+}$

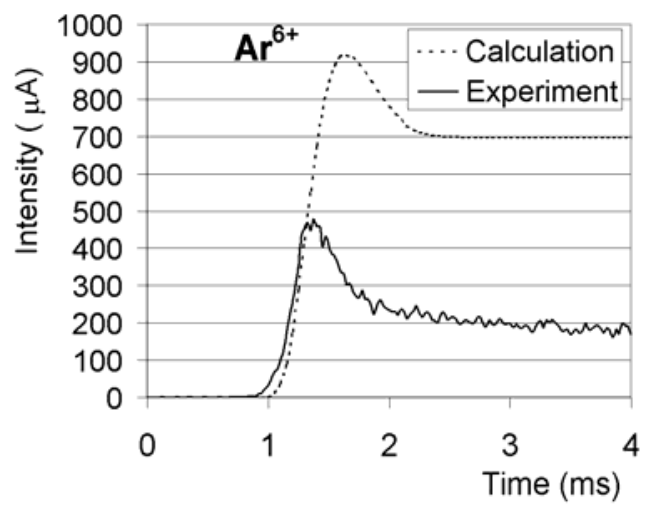

d) $\mathrm{Ar}^{6+}$ 


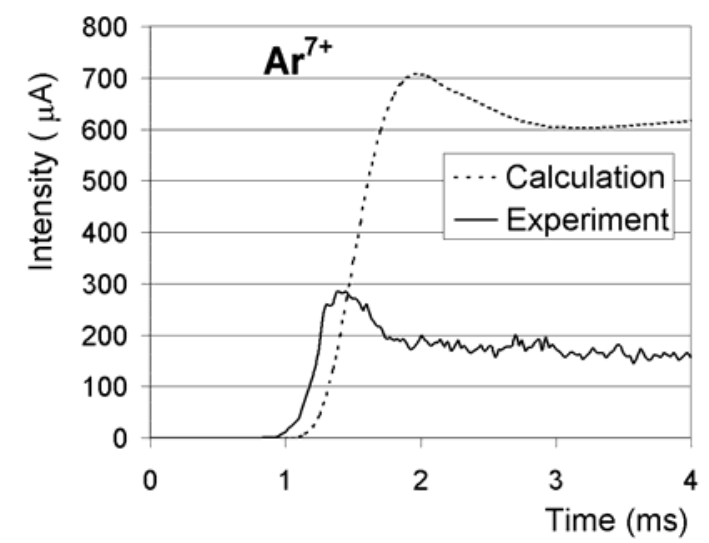

e) $\mathrm{Ar}^{7+}$



f) $\mathrm{Ar}^{8+}$

Fig. 4. Experimental argon pulses and their numerical fits for a) $\mathrm{Ar}^{2+}$, b) $\left.\mathrm{Ar}^{3+}, \mathrm{c}\right) \mathrm{Ar}^{4+}$, d) $\mathrm{Ar}^{6+}$, e) $\left.\mathrm{Ar}^{7+}, \mathrm{f}\right) \mathrm{Ar}^{8+}$. Calculated $<E>$ is also plotted on $\mathrm{c}$ ).

\section{B. Gaussian Fit of the Experimental PGW and Parameters Simulation}

In order to study the PGW peak characteristics, several parameters have been introduced for convenience based on the results of a Gaussian fit of the PGW peaks. The PGW parameters, presented in Fig. 5, are as follows: Imax is the peak maximum current, FWHM is the peak full width at half-maximum, TImax is the time to reach the maximum intensity ( $\mathrm{t}=0$ being microwave trigger) and T10 is the time to reach $10 \%$ of Imax. The PGW peak is not symmetric, so the fits were made using mainly the left part of the curve, ie for time $t \leq$ TImax.

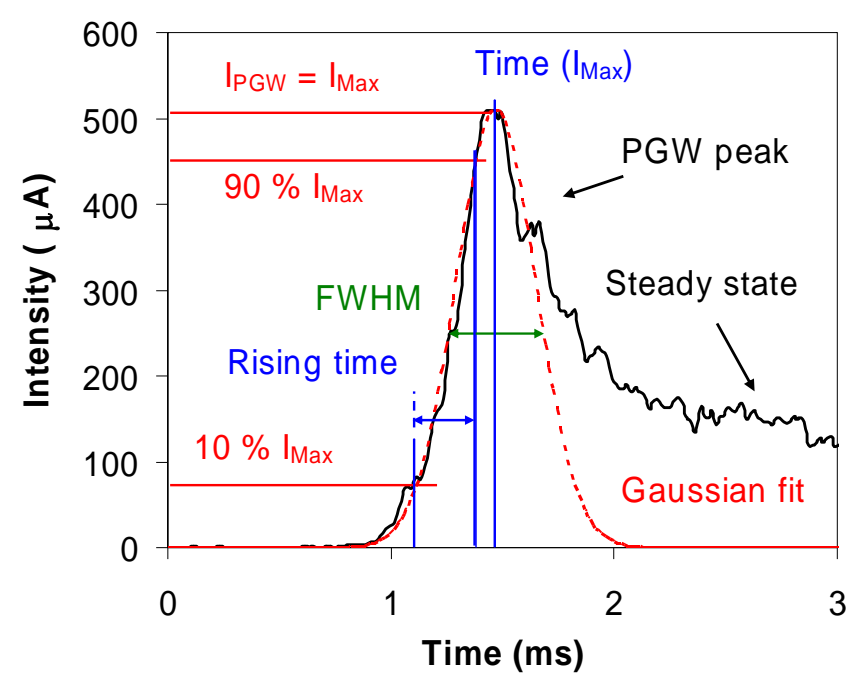

Fig. 5. Experimental PGW along with a Gaussian fit.

The variations of such PGW parameters as $T_{10}, T_{\text {Imax }}$, FWHM and Imax with the ion charge states are shown 
Fig. 6, in the case of the experimental conditions described in paragraph II (microwave power $=3100 \mathrm{~W})$.

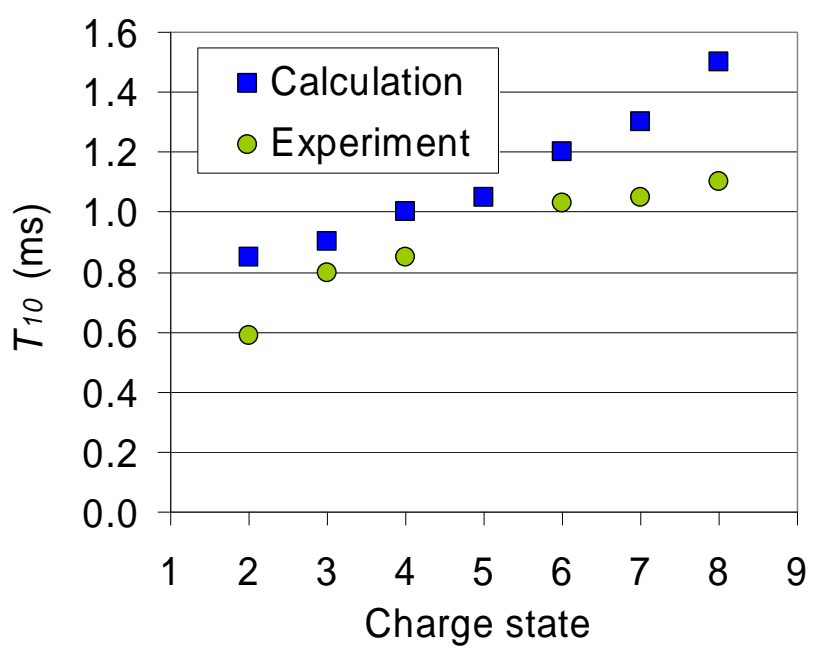

a) $T_{10}$

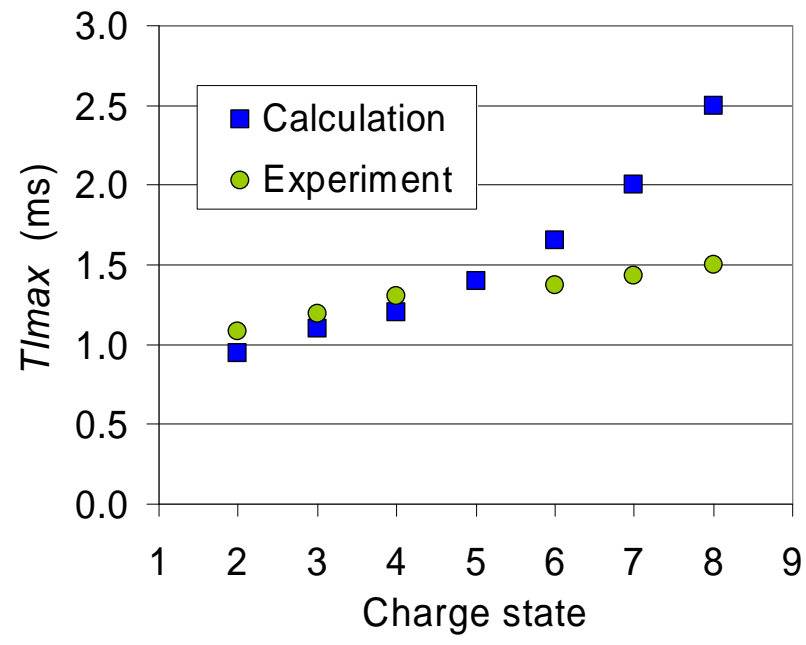

b) $T_{\operatorname{Imax}}$

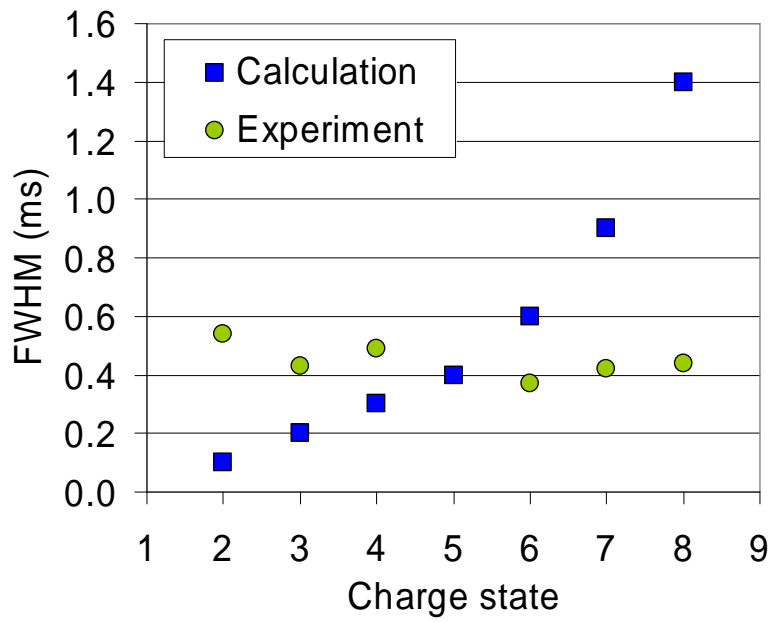

c) FWHM 


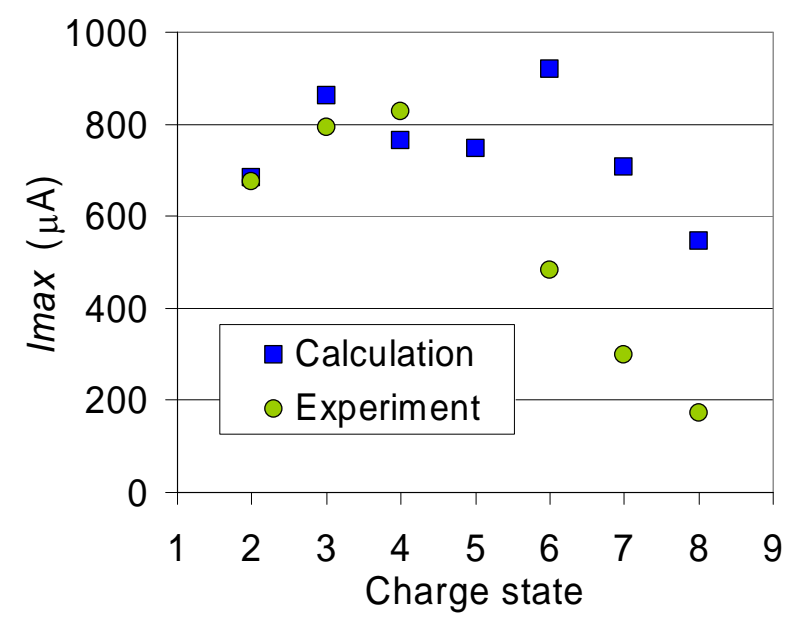

d) $I_{\max }$

Fig. 6. Preglow peaks characteristics for a microwave power of $3100 \mathrm{~W}(\mathrm{P}=16 \mathrm{~W} / \mathrm{cm} 2)$ : comparison between experimental and numerical fits for a) T10 b) TImax c) FWHM d) Imax.

To check the validity of the simulation of the peak intensities, the microwave power has been varied (three different levels of microwave power have been injected: $3000 \mathrm{~W}, 4500 \mathrm{~W}, 5000 \mathrm{~W}$ ) keeping all the other ECRIS parameters constant. The charge state dependence of $I_{\max }$ has been plotted Fig. 7 with the results of the numerical calculations (the $P$ values in the simulation were respectively $15 \mathrm{~W} / \mathrm{cm}^{2}, 22.5 \mathrm{~W} / \mathrm{cm}^{2}$, and $25 \mathrm{~W} / \mathrm{cm}^{2}$.

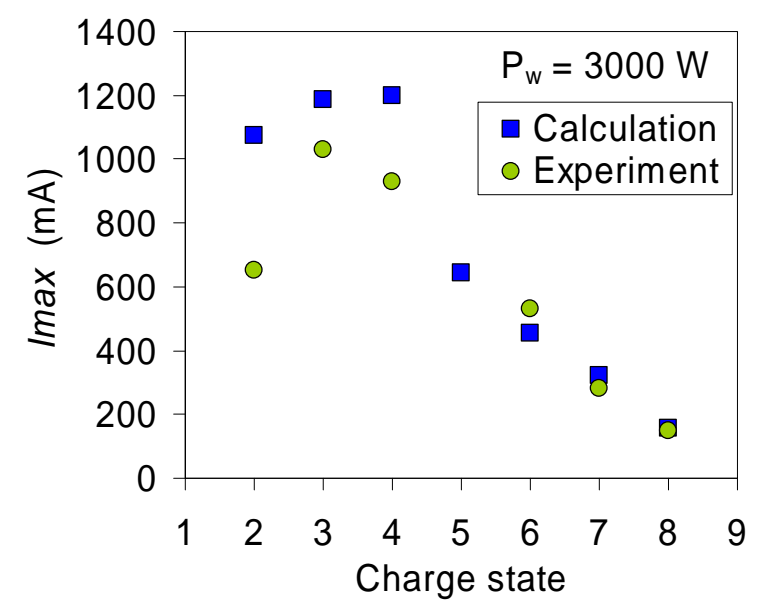

a) $3000 \mathrm{~W}\left(P=15 \mathrm{~W} / \mathrm{cm}^{2}\right)$

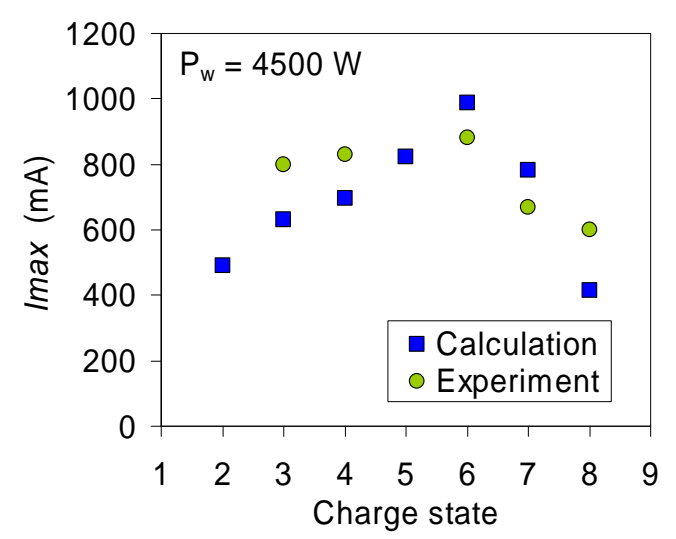

b) $4500 \mathrm{~W}\left(P=22.5 \mathrm{~W} / \mathrm{cm}^{2}\right)$ 


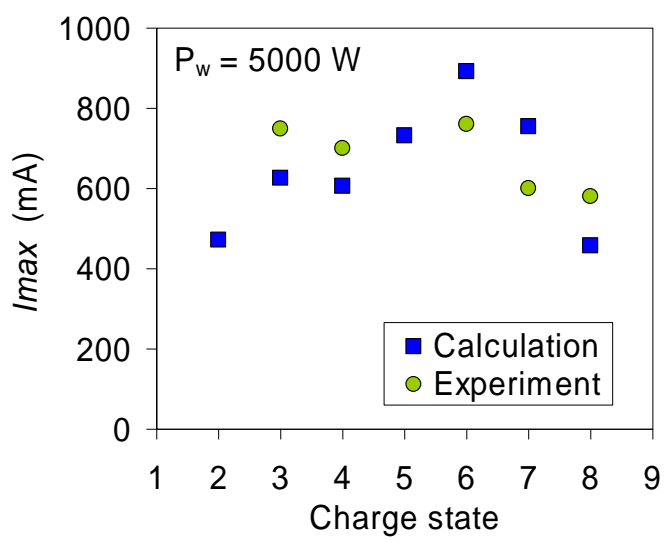

c) $5000 \mathrm{~W}\left(P=25 \mathrm{~W} / \mathrm{cm}^{2}\right)$

Fig. 7. Imax for different Ar charge states with increasing microwave power: a) $3000 \mathrm{~W}$ b) $4500 \mathrm{~W} \mathrm{c)} 5000 \mathrm{~W}$

It is visible on Fig. 6 and Fig. 7 that the proposed numerical model is able to predict the main PGW parameters dependences for charge states $q=2,3$ and 4 . A huge deviation appears for higher charge states, not due to charge exchange processes which were included in the simulation. The evolution of the $I_{\max }$ values obtained by the simulations for the consecutive charge states presents one or two maxima, they could be due to competitive effects that have not been yet identified by the authors. The general agreement between calculations and experimental results allows us to propose an explanation of the PGW phenomenon based on the results of the computer modeling. In Fig. 4-c) the $\mathrm{Ar}^{4+}$ PGW peak is presented together with the calculation of the average electron energy in the discharge. After $t \approx 1 \mathrm{~ms}$, the fall away of $\langle E>$ is connected with the plasma density overrunning the level where the EEDF transits from superadiabatic to Maxwellian. Further plasma density increase leads to a decrease of $\langle E>$. The longitudinal lifetime is decreasing as a result of the $\langle E>$ dropping which provides a fast plasma outlet from the ECRIS and the formation of a PGW peak.

The only experimental parameter we could not change during experiments was the microwave frequency, the PHOENIX-V2 ion source having only one microwave injection port. The relatively good agreement between experimental results and simulations, in the case of $\mathrm{Ar}^{4+}$, allows us to make a qualitative numerical estimation of the evolution of the PGW parameters with the microwave frequency. In this simulation, other parameters of modeling were chosen to maintain the same charge state distribution along the microwave frequency scale. It is visible on Fig. 8 that the PGW intensity is increasing and the time width of the signal is decreasing for higher microwave frequencies. The use of frequencies higher than $28 \mathrm{GHz}$ associated with higher microwave power injection will permit to increase the plasma density and then should permit to increase PGW efficiency for high charge states.

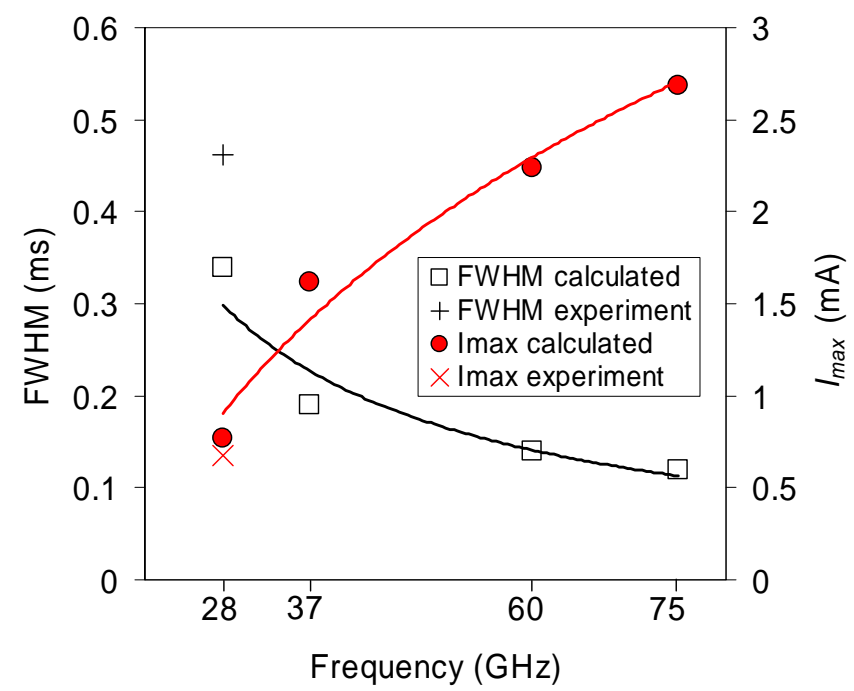

Fig. 8. Microwave frequency influence on PGW parameters 


\section{CONCLUSION}

To produce neutrino beams, the use of intense and pulsed accelerated radioactive ion beams is under evaluation for the Beta Beam project connected to the EURISOL facility. When using radioactive ion beams, efficiency is an important parameter, the work presented here does not evaluate nor measure the efficiency, however this work shows the possibility to produce intense pulsed beams about one order of magnitude below the expected intensities for the beta beam project and shows a potential increase of this intensity when using higher microwave frequencies. The authors expect that future study of the Preglow phenomenon under conditions of 60 GHz microwave ECR heating should effectively show the Preglow peak FWHM reduction, and an intensity increase leading to the expected intensity level.

\section{ACKNOWLEDGMENT}

The authors wish to pay tribute to Pr. Richard Geller who pushed us with all his strength to go forward in the PGW study.

\section{REFERENCES}

[1] P. Briand, R. Geller and G. Melin, "A newly designed ECR source for the lead injector of CERN," Nucl. Instrum. Meth. Phys. Res., Sec. A, Vol. 294(3), p. 673, Sept. 1990.

[2] P. Sortais, "Pulsed ECR ion source using the afterglow mode,"

Rev. Sci. Instrum., Vol. 63(4), pp. 2801-2805, Apr. 1992.

[3] C. Hill and K. Langbein, "Pulsed ECR source in afterglow operation at CERN,"

Rev. Sci. Instrum., Vol. 67(3), pp. 1328-1330, Mar. 1996.

[4] http://beta-beam.web.cern.ch/beta-beam/task/index.asp

[5] P. Sortais, J.L. Bouly, J.-C. Curdy, T. Lamy, P. Sole, T. Thuillier, J.L. Vieux-Rochaz, D. Voulot, "ECRIS development for stable and radioactive pulsed beams,"

Rev. Sci. Instrum., Vol. 75(5), pp. 1610-1612, May 2004.

[6] T. Thuillier, J.L. Bouly, J.C. Curdy, E. Froidefond, T. Lamy, C. Peaucelle, P. Sole, P. Sortais, J.L. Vieux-

Rochaz, D. Voulot, "High Intensity Ion Beams Prospects for Accelerators with PHOENIX 28 GHz,"

in Proc. 8th European Particle Accelerator Conf., Paris, 2002, pp. 1744-1746.

[7] Y. Bykov, G. Denisov, A. Eremeev, V. Gorbatushkov, V. Kurkin, G. Kalynova, V. Kholoptsev, A. Luchinin, and I. Plotnikov, " $28 \mathrm{GHz} 10 \mathrm{~kW}$ gyrotron system for electron cyclotron resonance ion source,"

Rev. Sci. Instrum., Vol. 75(5), pp. 1437-1439 May 2004.

[8] T. Thuillier, T. Lamy, L. Latrasse, I. V. Izotov, A. V. Sidorov, V. A. Skalyga, V. G. Zorin, M. Marie-Jeanne, "Study of pulsed electron cyclotron resonance ion source plasma near breakdown: The Preglow,"

Rev. Sci. Instrum., Vol. 79(2), 02A314-02A316, Feb. 2008.

[9] V.E. Semenov, V.A. Skalyga, V.G. Zorin, "Scaling for ECR Sources of Multicharged Ions with Pumping at Frequencies from 10 to $100 \mathrm{GHz}$,"

Rev. Sci. Instrum., Vol. 73(2), pp. 635-637, Feb. 2002.

[10] E.V. Suvorov and M.D. Tokman, "Theory of microwave breakdown of low-density gas at electron cyclotron resonance in magnetic mirror systems,"

Sov. J. Plasma Phys. Vol. 15, p. 540, 1989, ("K teorii SVCh proboja razrezhennogo gaza v adiabaticheskoj magnitnoj lovushke pri elektronno-tsiklotronnom rezonanse," Fiz. Plazmy 15, 934, 1989).

[11] Edgell D.H., Kim J.S., Bogatu I.N., Pardo R.C., Vondrasek R.C. "Modeling of electron cyclotron resonance ion source plasmas,"

in Proc. Part. Accel. Conf., Chicago, June 18-22, 2001, Vol. 3, pp. 2135-2137.

[12] V.M. Povyshev, A.A. Sadovoy, V.P. Shevelko, G.D. Shirkov, E.G Vasina, V.V. Vatulin, "Electron impact ionization cross-sections of $\mathrm{H}, \mathrm{He}, \mathrm{N}, \mathrm{O}, \mathrm{Ar}, \mathrm{Xe}, \mathrm{Au}, \mathrm{Pb}$ atoms and their ions in the electron energy range from the threshold up to 200-keV,"

JINR-E9-2001-148, [online]. Available: http://www1.jinr.ru/Preprints/2001/e9-2001-148.pdf

[13] V. P. Pastukhov, "Collisional losses of electron from an adiabatic trap in a plasma with a positive potential," Nucl. Fusion, Vol. 14(1), pp. 3-6, Jan.1974.

[14] H.I. West, "Calculations of Ion Charge-State Distribution in ECR ion Sources"

Lawrence Livermore National Lab., CA (USA). Report UCRL-53391, Dec. 1982.

[15] T.D. Rognlien, T.A. Cutler, "Transition from Pastukhov to collisional confinement in a magnetic and electrostatic well"

Nucl. Fusion, Vol. 20(8), pp. 1003-1011, 1980.

[16] Mirnov V.V., Ryutov D.D., "Linear gasdynamic system for plasma confinement,"

Sov. Tekh. Phys. Lett., Vol. 5(6), pp. 279-280, Jun. 1979.

"Gazodinamicheskaja linejnaya lovushka dlya uderzhanija plazmy,"

Pisma v Zhurnal Theknicheskoj Fiziki, Vol.5, p. 678, 1979. 
[17] S.V. Golubev, S.V. Razin, V.E. Semenov, A. N. Smirnov, A.V. Vodopyanov, V.G. Zorin, "Formation of multicharged ions and plasma stability at quasigasdynamic plasma confinement in a mirror magnetic trap," Rev. Sci. Instrum., Vol. 71(2), pp. 669-671, Feb. 2000. 